\title{
The n-Hosoya Polynomials of the Square of a Path and of a Cycle
}

\author{
Ahmed M. Ali \\ ahmedgraph@uomousl.edu.iq \\ Department of Mathematics \\ College of Computer Science and Mathematics \\ University of Mosul, Mosul, Iraq
}

\section{Received on: 02/06/2013}

Accepted on: 16/09/2013

\section{ABSTRACT}

The n-Hosoya polynomial of a connected graph $\mathrm{G}$ of order $\mathrm{t}$ is defined by: $H_{n}(G ; x)=\sum_{k=0}^{\delta_{n}} C_{n}(G, k) x^{k}$, Where, $\mathrm{C}_{\mathrm{n}}(\mathrm{G}, \mathrm{k})$ is the number of pairs $(\mathrm{v}, \mathrm{S})$, in which $|S|=n-1, \quad 3 \leq n \leq t, \quad v \in V(G), \quad S \subseteq V(G), \quad$ such that $\mathrm{d}_{\mathrm{n}}(\mathrm{v}, \mathrm{S})=\mathrm{k}, \quad$ for each $0 \leq k \leq \delta_{n}=\operatorname{diam}_{n}(G)$.

In this paper, we find the n-Hosoya polynomial of the square of a path and of the square of a cycle. Also, the n-diameter and n-Wiener index of each of the two graphs are determined.

Keyword: n-diameter, n-Hosoya polynomial, n-Wiener index, path square and cycle square.

$$
\begin{aligned}
& \text { متعددات حدود هوسويا-n لمربعي الدرب واللارة } \\
& \text { أحدد عحا علمي } \\
& \text { قسم الرياضيات، كلية علوم الحاسوب والرياضيات }
\end{aligned}
$$

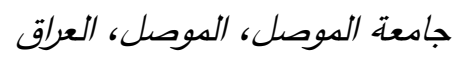

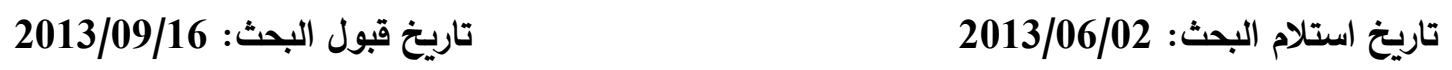

$$
\begin{aligned}
& \text { الملخص } \\
& \text { تعرف متعددة حدود هوسويا-n لبيان متصل G من الرتبة t على أنها: } H_{n}(G ; x)=\sum_{k=0}^{\delta_{n}} C_{n}(G, k) x^{k}
\end{aligned}
$$

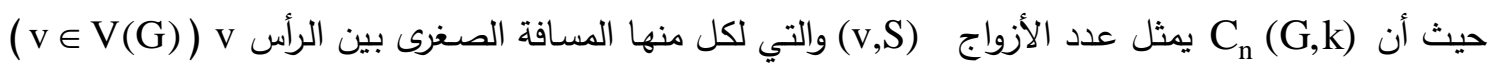

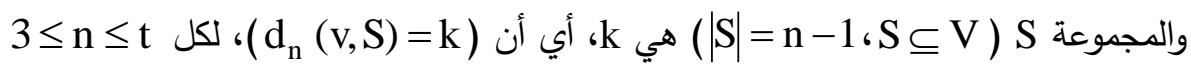

$$
\begin{aligned}
& \text {. } 0 \leq \mathrm{k} \leq \delta_{\mathrm{n}}=\operatorname{diam}_{\mathrm{n}}(\mathrm{G}) \text { و } \\
& \text { في هذا البحث تم إيجاد متعددة حدود هوسويا-n لكل من مربع الدرب ومربع الدارة، وكذلك تم تحديد القطر - }
\end{aligned}
$$

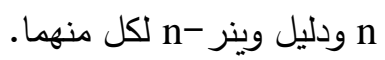

$$
\begin{aligned}
& \text { الكلمات المفتاحية: القطر -n ، متعددات حدود هوسويا - n، دليل وينر - n ،مربع الدرب ، مربع الدارة. }
\end{aligned}
$$




\section{Introduction:}

The n-distance [1] in a connected graph $\mathrm{G}=(\mathrm{V}, \mathrm{E})$ of order $\mathrm{t}$ is the minimum distance from a singleton, $\mathrm{v} \in \mathrm{V}$ to an (n-1)-subset $\mathrm{S}, \mathrm{S} \subseteq \mathrm{V}, 3 \leq \mathrm{n} \leq \mathrm{t}$, that is , $\mathrm{d}_{\mathrm{n}}(\mathrm{v}, \mathrm{S})=\min \{\mathrm{d}(\mathrm{v}, \mathrm{u}): \mathrm{u} \in \mathrm{S}\}, 3 \leq \mathrm{n} \leq \mathrm{t}$.

It is clear that $\mathrm{d}_{\mathrm{n}}(\mathrm{v}, \mathrm{S})=0$; when $\mathrm{v} \in \mathrm{S}$, $\mathrm{d}_{\mathrm{n}}(\mathrm{v}, \mathrm{S}) \geq 1$; when $\mathrm{v} \notin \mathrm{S}$.

The $\underline{n-W i e n e r ~ i n d e x ~ o f ~ a ~ c o n n e c t e d ~ g r a p h ~} G=(V, E)$ is the sum of the minimum distances of all pairs $(\mathrm{v}, \mathrm{S})$ in the graph $\mathrm{G}$, that is:

$$
\mathrm{W}_{\mathrm{n}}(\mathrm{G})=\sum_{\substack{(\mathrm{v}, \mathrm{S}),|\mathrm{S}|=\mathrm{n}-1 \\ \mathrm{v} \in \mathrm{V}, \mathrm{S} \subseteq \mathrm{V}}} \mathrm{d}_{\mathrm{n}}(\mathrm{v}, \mathrm{S}), 3 \leq \mathrm{n} \leq \mathrm{t} .
$$

The $\underline{n-d i a m e t e r ~ o f ~} \mathrm{G}$ is defined by:

$$
\operatorname{diam}_{\mathrm{n}} \mathrm{G}=\max \left\{\mathrm{d}_{\mathrm{n}}(\mathrm{v}, \mathrm{S}): \mathrm{v} \in \mathrm{V}(\mathrm{G}),|\mathrm{S}|=\mathrm{n}-1, \mathrm{~S} \subseteq \mathrm{V}(\mathrm{G})\right\} .
$$

Now, let $C_{n}(G, k)$ be the number of pairs $(v, S),|S|=n-1,3 \leq n \leq t, v \in V$, $\mathrm{S} \subseteq \mathrm{V}$, such that $\mathrm{d}_{\mathrm{n}}(\mathrm{v}, \mathrm{S})=\mathrm{k}$, for each $0 \leq \mathrm{k} \leq \delta_{\mathrm{n}}=\operatorname{diam}_{\mathrm{n}}(\mathrm{G})$, then the $\underline{\mathrm{n}-\text { Hosoya }}$ polynomial of $\mathrm{G}$ is defined by:

$$
\mathrm{H}_{\mathrm{n}}(\mathrm{G} ; \mathrm{x})=\sum_{\mathrm{k}=0}^{\delta_{\mathrm{n}}} \mathrm{C}_{\mathrm{n}}(\mathrm{G}, \mathrm{k}) \mathrm{x}^{\mathrm{k}} \text {. }
$$

We can obtain the $n$-Wiener index of $G$ from the $n$-Hosoya polynomial of $G$ as follows:

$$
\mathrm{W}_{\mathrm{n}}(\mathrm{G})=\left.\frac{\mathrm{d}}{\mathrm{dx}} \mathrm{H}_{\mathrm{n}}(\mathrm{G} ; \mathrm{x})\right|_{\mathrm{x}=1}=\sum_{\mathrm{k}=1}^{\delta_{\mathrm{n}}} \mathrm{kC}_{\mathrm{n}}(\mathrm{G}, \mathrm{k}) \text {. }
$$

For a vertex $v$ of a connected graph $G$, let $C_{n}(v, G, k)$ be the number of (n-1)-subsets $S$ of vertices of $G$ such that $d_{n}(v, S)=k$, for $n \geq 3,0 \leq k \leq \delta_{n}$. The $\underline{n-H o s o y a}$ polynomial of the vertex $\mathrm{v}$, denoted by $\mathrm{H}_{\mathrm{n}}(\mathrm{v}, \mathrm{G} ; \mathrm{x})$, is defined as:

$$
\mathrm{H}_{\mathrm{n}}(\mathrm{v}, \mathrm{G} ; \mathrm{x})=\sum_{\mathrm{k} \geq 0} \mathrm{C}_{\mathrm{n}}(\mathrm{v}, \mathrm{G}, \mathrm{k}) \mathrm{x}^{\mathrm{k}} \text {. }
$$

It is clear that for all $\mathrm{k} \geq 0$,

$$
\sum_{v \in V(G)} C_{n}(v, G, k)=C_{n}(G, k)
$$

and

$$
\sum_{v \in V(G)} H_{n}(v, G ; x)=H_{n}(G ; x) \text {. }
$$

For more information about these concepts, see the References $[1,2,5,6]$.

The next lemma will be used in proving our results.

Lemma 1.1:[1] Let $v$ be any vertex of a connected graph $G$. If there are $r$ vertices of distance $\mathrm{k} \geq 1$ from $\mathrm{v}$, and there are $\mathrm{s}$ vertices of distance more than $\mathrm{k}$ from $\mathrm{v}$, then, for $\mathrm{n} \geq 3$, 


$$
\mathrm{C}_{\mathrm{n}}(\mathrm{v}, \mathrm{G}, \mathrm{k})=\left(\begin{array}{l}
\mathrm{r}+\mathrm{s} \\
\mathrm{n}-1
\end{array}\right)-\left(\begin{array}{c}
\mathrm{s} \\
\mathrm{n}-1
\end{array}\right) .
$$

Definition 1.2: Let $G$ be a connected non-trivial graph . The square $G^{2}$ of the graph $G$ , introduced by Harary and Ross [7], has $V\left(G^{2}\right)=V(G)$ with $u, v$ adjacent in $G^{2}$, whenever $1 \leq \mathrm{d}_{\mathrm{G}}(\mathrm{u}, \mathrm{v}) \leq 2$.

Notice that the square of complete graph, star graph, wheel graph, complete bipartite graph are complete graphs.

In $[1,2,3,4]$, the n-Hosoya polynomials for many special graphs and many compound graphs are obtained. In this paper, we continue such works by obtaining the n-Hosoya polynomials of the square of paths and cycles.

\section{The n-Hosoya Polynomial of the Square of a Path:}

In this section, we obtained the n-Hosoya polynomial of the square $\mathrm{P}_{\mathrm{t}}^{2}$ of a path $\mathrm{P}_{t}$ of order $\mathrm{t}$. We shall consider two main cases of $\mathrm{P}_{t}^{2}$ according to the parity of $t$.

First Case : Even $t, t=2 r, r \geq 2$.

Let $\mathrm{P}_{\mathrm{t}}: \mathrm{u}_{1}, \mathrm{u}_{2}, \mathrm{u}_{3}, \ldots, \mathrm{u}_{\mathrm{t}}$, then $\mathrm{P}_{\mathrm{t}}^{2}$ is shown in Fig.2.1, and by relabeling its vertices, we have Fig. 2.2 for $\mathrm{P}_{2 \mathrm{r}}^{2}$.

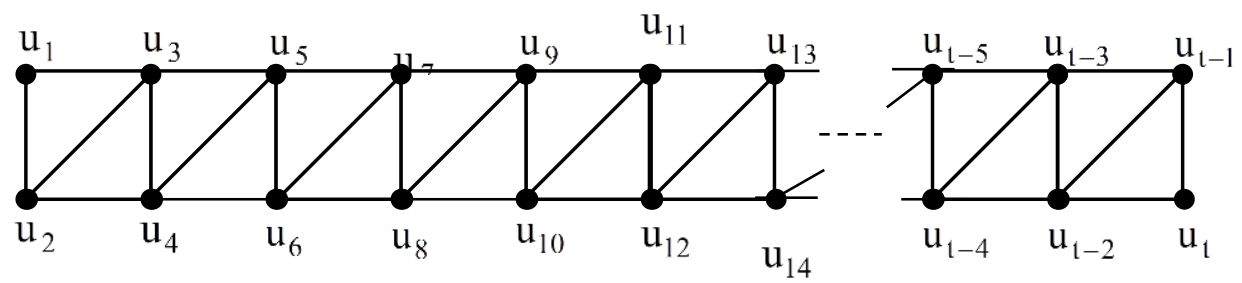

Fig. (2.1). The Path Square $P_{t}^{2}$

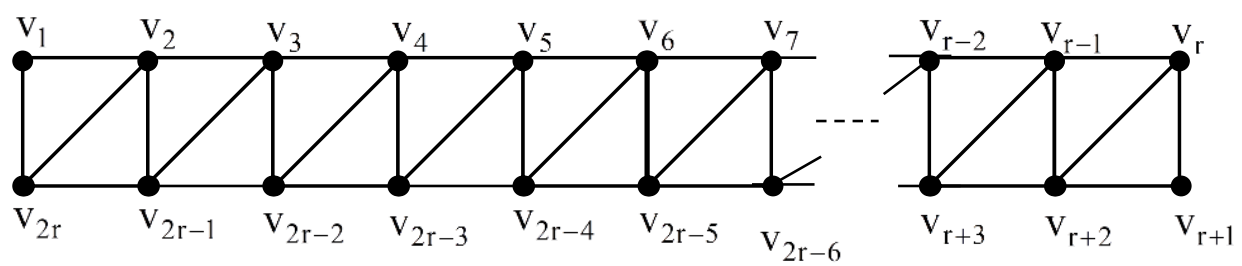

Fig. (2.2). The Path Square $\mathrm{P}_{2 \mathrm{r}}^{2}$

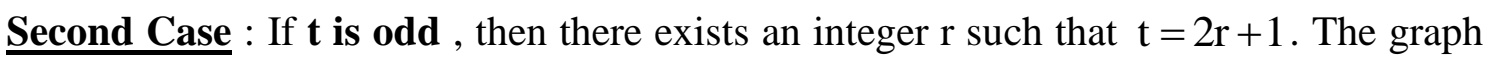
$\mathrm{P}_{\mathrm{t}}^{2}$ is shown in Fig.2-3.

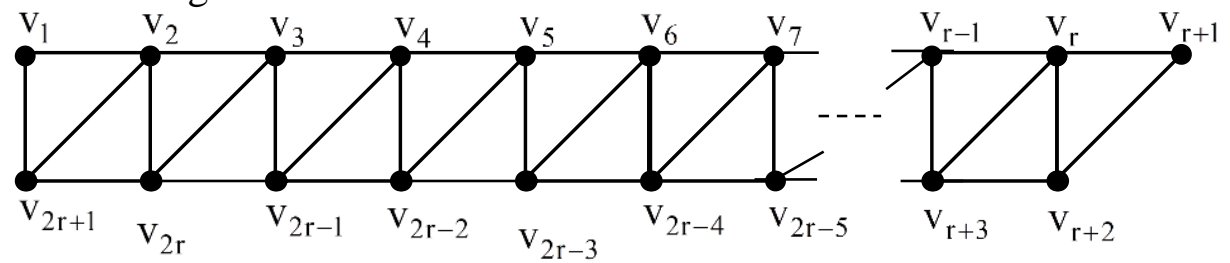

Fig. (2.3). The Path Square $\mathrm{P}_{2 \mathrm{r}+1}^{2}$. 
Theorem 2.1: For $\mathrm{t} \geq 5$ and $\mathrm{n} \geq 2$, let $\mathrm{r}=\left\lfloor\frac{\mathrm{t}}{2}\right\rfloor$, then, $\operatorname{diam}_{n}\left(P_{t}^{2}\right)= \begin{cases}r+1-\left\lceil\frac{n}{2}\right\rceil, & \text { for even } t \\ r+1-\left\lfloor\frac{n}{2}\right\rfloor, & \text { for odd } t\end{cases}$

\section{Proof:}

(1). Let $t$ be even, then $t=2 r$.

From Fig.2.2, we notice that $\operatorname{diam}\left(\mathrm{P}_{2 \mathrm{r}}^{2}\right)=\mathrm{d}\left(\mathrm{v}_{1}, \mathrm{v}_{\mathrm{r}+1}\right)=\mathrm{r}$, then $\operatorname{diam}_{\mathrm{n}}\left(\mathrm{P}_{2 \mathrm{r}}^{2}\right)=\mathrm{d}_{\mathrm{n}}\left(\mathrm{v}_{1}, \mathrm{~S}\right)$, $\mathrm{n} \geq 2$, where $\mathrm{S}$ consists of the first $\mathrm{n}-1$ vertices from the sequence $\left\{\mathrm{v}_{\mathrm{r}+1} ; \mathrm{v}_{\mathrm{r}}, \mathrm{v}_{\mathrm{r}+2} ; \mathrm{v}_{\mathrm{r}-1}, \mathrm{v}_{\mathrm{r}+3} ; \mathrm{v}_{\mathrm{r}-2}, \mathrm{v}_{\mathrm{r}+4} ; \ldots ; \mathrm{v}_{2}, \mathrm{v}_{2 \mathrm{r}}\right\}$.

Thus, if $\mathrm{n}$ is even, then

$\mathrm{S}=\left\{\mathrm{v}_{\mathrm{r}+1}\right\}, \mathrm{n}=2$,

$\mathrm{S}=\left\{\mathrm{v}_{\mathrm{r}+1}, \mathrm{v}_{\mathrm{r}}, \mathrm{v}_{\mathrm{r}+2} ; \mathrm{v}_{\mathrm{r}-1}, \mathrm{v}_{\mathrm{r}+3} ; \mathrm{v}_{\mathrm{r}-2}, \mathrm{v}_{\mathrm{r}+4} ; \ldots ; \mathrm{v}_{\mathrm{r}+2-\frac{\mathrm{n}}{2}}, \mathrm{v}_{\mathrm{r}+\frac{\mathrm{n}}{2}}\right\}, \mathrm{n}=4,6,8, \ldots, 2 \mathrm{r}$.

So, $\mathrm{d}_{\mathrm{n}}\left(\mathrm{v}_{1}, \mathrm{~S}\right)=\mathrm{r}+1-\frac{\mathrm{n}}{2}$.

If $\mathrm{n}$ is odd, then

$\mathrm{S}=\left\{\mathrm{v}_{\mathrm{r}+1}, \mathrm{v}_{\mathrm{r}} ; \mathrm{v}_{\mathrm{r}+2}, \mathrm{v}_{\mathrm{r}-1} ; \mathrm{v}_{\mathrm{r}+3} ; \mathrm{v}_{\mathrm{r}-2} ; \ldots ; \mathrm{v}_{\mathrm{r}+\frac{\mathrm{n}-1}{2}}, \mathrm{v}_{\mathrm{r}+1-\frac{\mathrm{n}-1}{2}}\right\}, \mathrm{n}=3,5,7, \ldots, 2 \mathrm{r}-1$.

So, $\mathrm{d}_{\mathrm{n}}\left(\mathrm{v}_{1}, \mathrm{~S}\right)=\mathrm{r}+1-\frac{\mathrm{n}+1}{2}$.

Therefore, $\operatorname{diam}_{\mathrm{n}}\left(\mathrm{P}_{2 \mathrm{r}}^{2}\right)=\mathrm{r}+1-\left\lceil\frac{\mathrm{n}}{2}\right\rceil$, for all $\mathrm{n} \geq 2$.

(2). Let $\mathrm{t}$ be odd, then $\mathrm{t}=2 \mathrm{r}+1$.

From Fig.2.3, we notice that $\operatorname{diam}\left(\mathrm{P}_{2 \mathrm{r}+1}^{2}\right)=\mathrm{d}\left(\mathrm{v}_{1}, \mathrm{v}_{\mathrm{r}+1}\right)=\mathrm{r},\left(\right.$ or $\mathrm{d}\left(\mathrm{v}_{1}, \mathrm{v}_{\mathrm{r}+2}\right)$, or $\left.\mathrm{d}\left(\mathrm{v}_{2 \mathrm{r}+1}, \mathrm{v}_{\mathrm{r}+1}\right)\right)$, then $\operatorname{diam}_{\mathrm{n}}\left(\mathrm{P}_{2 \mathrm{r}+1}^{2}\right)=\mathrm{d}_{\mathrm{n}}\left(\mathrm{v}_{1}, \mathrm{~S}\right),|\mathrm{S}|=\mathrm{n}-1, \mathrm{n} \geq 2$, where $\mathrm{S}$ consists of the first $\mathrm{n}-1$ vertices from the sequence $\left\{\mathrm{V}_{\mathrm{r}+1}, \mathrm{~V}_{\mathrm{r}+2} ; \mathrm{V}_{\mathrm{r}}, \mathrm{V}_{\mathrm{r}+3} ; \mathrm{V}_{\mathrm{r}-1}, \mathrm{~V}_{\mathrm{r}+4} ; \ldots ; \mathrm{V}_{2}, \mathrm{v}_{2 \mathrm{r}+1}\right\}$.

Thus, if $\mathrm{n}$ is odd, then,

$\mathrm{S}=\left\{\mathrm{v}_{\mathrm{r}+1}, \mathrm{v}_{\mathrm{r}+2} ; \mathrm{v}_{\mathrm{r}}, \mathrm{v}_{\mathrm{r}+3} ; \mathrm{v}_{\mathrm{r}-1}, \mathrm{v}_{\mathrm{r}+4} ; \ldots ; \mathrm{v}_{\mathrm{r}+2-\frac{\mathrm{n}-1}{2}}, \mathrm{v}_{\mathrm{r}+1+\frac{\mathrm{n}-1}{2}}\right\}, \mathrm{n}=3,5,7, \ldots, 2 \mathrm{r}+1$.

So, $\mathrm{d}_{\mathrm{n}}\left(\mathrm{v}_{1}, \mathrm{~S}\right)=\mathrm{d}\left(\mathrm{v}_{1}, \mathrm{v}_{\mathrm{r}+2-\frac{\mathrm{n}-1}{2}}\right)=\mathrm{r}+1-\frac{\mathrm{n}-1}{2}$.

If $\mathrm{n}$ is even, then,

$\mathrm{S}=\left\{\mathrm{v}_{\mathrm{r}+1}, \mathrm{v}_{\mathrm{r}+2} ; \mathrm{v}_{\mathrm{r}}, \mathrm{v}_{\mathrm{r}+3} ; \mathrm{v}_{\mathrm{r}-1}, \mathrm{v}_{\mathrm{r}+4} ; \ldots ; \mathrm{v}_{\mathrm{r}+2-\frac{\mathrm{n}}{2}}, \mathrm{v}_{\mathrm{r}+1+\frac{\mathrm{n}}{2}}\right\}, \mathrm{n}=4,6,8, \ldots, 2 \mathrm{r}$.

So, $\mathrm{d}_{\mathrm{n}}\left(\mathrm{v}_{1}, \mathrm{~S}\right)=\mathrm{d}\left(\mathrm{v}_{1}, \mathrm{v}_{\mathrm{r}+2-\frac{\mathrm{n}}{2}}\right)=\mathrm{r}+1-\frac{\mathrm{n}}{2}$.

Therefore ,

$\operatorname{diam}_{\mathrm{n}}\left(\mathrm{P}_{2 \mathrm{r}+1}^{2}\right)=\mathrm{r}+1-\left\lfloor\frac{\mathrm{n}}{2}\right\rfloor$, for all $\mathrm{n} \geq 2 . \quad \#$ 
Remark : Throughout this work, we assume that $\left(\begin{array}{l}a \\ b\end{array}\right)=0$, if $a<b$.

Theorem 2.2: For any $n \geq 3$, the $n$-Hosoya polynomial of $P_{t}^{2}, t \geq 6$, is given by:

$\mathrm{H}_{\mathrm{n}}\left(\mathrm{P}_{\mathrm{t}}^{2} ; \mathrm{x}\right)=\sum_{\mathrm{k}=0}^{\delta_{\mathrm{n}}} \mathrm{C}_{\mathrm{n}}\left(\mathrm{P}_{\mathrm{t}}^{2}, \mathrm{k}\right) \mathrm{x}^{\mathrm{k}}$,

Where, $\delta_{\mathrm{n}}=\operatorname{diam}_{\mathrm{n}}\left(\mathrm{P}_{\mathrm{t}}^{2}\right)$,

$\mathrm{C}_{\mathrm{n}}\left(\mathrm{P}_{\mathrm{t}}^{2}, 0\right)=\mathrm{t}\left(\begin{array}{l}\mathrm{t}-1 \\ \mathrm{n}-2\end{array}\right)$,

$\mathrm{C}_{\mathrm{n}}\left(\mathrm{P}_{\mathrm{t}}^{2}, 1\right)=\mathrm{t}\left(\begin{array}{l}\mathrm{t}-1 \\ \mathrm{n}-1\end{array}\right)-2\left[\left(\begin{array}{l}\mathrm{t}-3 \\ \mathrm{n}-1\end{array}\right)+\left(\begin{array}{l}\mathrm{t}-4 \\ \mathrm{n}-1\end{array}\right)\right]-(\mathrm{t}-4)\left(\begin{array}{l}\mathrm{t}-5 \\ \mathrm{n}-1\end{array}\right)$,

$\mathrm{C}_{\mathrm{n}}\left(\mathrm{P}_{\mathrm{t}}^{2}, \mathrm{k}\right)=2\left[\left(\begin{array}{c}\mathrm{t}-2 \mathrm{k} \\ \mathrm{n}-1\end{array}\right)+\left(\begin{array}{c}\mathrm{t}-2 \mathrm{k}+1 \\ \mathrm{n}-1\end{array}\right)\right]+(\mathrm{t}-4 \mathrm{k}+2)\left(\begin{array}{c}\mathrm{t}-4 \mathrm{k}+3 \\ \mathrm{n}-1\end{array}\right)$

$$
-2 \sum_{\mathrm{i}=0}^{2}\left(\begin{array}{c}
\mathrm{t}-4 \mathrm{k}+\mathrm{i} \\
\mathrm{n}-1
\end{array}\right)-(\mathrm{t}-4 \mathrm{k})\left(\begin{array}{c}
\mathrm{t}-4 \mathrm{k}-1 \\
\mathrm{n}-1
\end{array}\right), 2 \leq \mathrm{k} \leq\left\lfloor\frac{\delta_{\mathrm{n}}}{2}\right\rfloor
$$

$\mathrm{C}_{\mathrm{n}}\left(\mathrm{P}_{\mathrm{t}}^{2}, \mathrm{k}\right)=2\left[\left(\begin{array}{c}\mathrm{t}-2 \mathrm{k} \\ \mathrm{n}-1\end{array}\right)+\left(\begin{array}{c}\mathrm{t}-2 \mathrm{k}+1 \\ \mathrm{n}-1\end{array}\right)\right],\left\lfloor\frac{\delta_{\mathrm{n}}}{2}\right\rfloor+1 \leq \mathrm{k} \leq \delta_{\mathrm{n}}$.

Proof: It is clear that $C_{n}\left(P_{t}^{2}, 0\right)=t\left(\begin{array}{l}t-1 \\ n-2\end{array}\right)$.

From Fig.2.2, we notice that in $\mathrm{P}_{\mathrm{t}}^{2}$, there are two vertices of degree 2, two vertices of degree 3 , and $\mathrm{t}-4$ vertices of degree 4 . Thus, using formula (1.4.5) in [1], we obtain (2.2.2).

For each vertex $\mathrm{w}$ and given $\mathrm{k}$, let

$\mathrm{S}_{1}(\mathrm{w}, \mathrm{k})=\{\mathrm{v} \in \mathrm{V}: \mathrm{d}(\mathrm{w}, \mathrm{v})=\mathrm{k}\}$,

$\mathrm{S}_{2}(\mathrm{w}, \mathrm{k})=\{\mathrm{v} \in \mathrm{V}: \mathrm{d}(\mathrm{w}, \mathrm{v})>\mathrm{k}\}$.

$\underline{\text { First }}$, we shall prove (2.2.3) and (2.2.4) for even $\mathbf{t}$, assuming $t=2 r, r \geq 4$. It is clear, from Fig. 2.2, that for $n \geq 3$,

$$
\mathrm{C}_{\mathrm{n}}\left(\mathrm{v}_{\mathrm{i}}, \mathrm{P}_{\mathrm{t}}^{2}, \mathrm{k}\right)=\mathrm{C}_{\mathrm{n}}\left(\mathrm{v}_{\mathrm{i}+\mathrm{r}}, \mathrm{P}_{\mathrm{t}}^{2}, \mathrm{k}\right) \text {, }
$$

for $\mathrm{i}=1,2, \ldots, \mathrm{r}$. Therefore, for $2 \leq \mathrm{k} \leq \delta_{\mathrm{n}}$,

$\mathrm{C}_{\mathrm{n}}\left(\mathrm{P}_{2 \mathrm{r}}^{2}, \mathrm{k}\right)=2 \sum_{\mathrm{i}=1}^{\mathrm{r}} \mathrm{C}_{\mathrm{n}}\left(\mathrm{v}_{\mathrm{i}}, \mathrm{P}_{2 \mathrm{r}}^{2}, \mathrm{k}\right)$.

Now, let $2 \leq \mathrm{k} \leq\left\lfloor\frac{\delta_{\mathrm{n}}}{2}\right\rfloor$, in which $\delta_{\mathrm{n}}$ is determined by Theorem 2.1, that is

$\delta_{\mathrm{n}}=\mathrm{r}+1-\left\lceil\frac{\mathrm{n}}{2}\right\rceil$.

Since, $\mathrm{n} \geq 3$, then $\delta_{\mathrm{n}} \leq \mathrm{r}-1$, for $\mathrm{r} \geq 4$. 
But, in proving (2.2.3), we assume that $\delta_{\mathrm{n}} \geq 4$.

According to the given value of $\mathrm{k}$, we partition $\left\{\mathrm{v}_{1}, \mathrm{v}_{2}, \ldots, \mathrm{v}_{\mathrm{r}}\right\}$ into the following four cases:

(1). For $\mathrm{i}=1,2, \ldots, \mathrm{k}$, we notice, from Fig. 2.2, that:

$\mathrm{S}_{1}\left(\mathrm{v}_{\mathrm{i}}, \mathrm{k}\right)=\left\{\mathrm{v}_{\mathrm{i}+\mathrm{k}}, \mathrm{v}_{2 \mathrm{r}+2-\mathrm{i}-\mathrm{k}}\right\}$

$\mathrm{S}_{2}\left(\mathrm{v}_{\mathrm{i}}, \mathrm{k}\right)=\mathrm{V}\left(\mathrm{P}_{2 \mathrm{r}}^{2}\right)-\left\{\mathrm{v}_{1}, \mathrm{v}_{2}, \ldots, \mathrm{v}_{\mathrm{i}+\mathrm{k}}, \mathrm{v}_{2 \mathrm{r}+2-\mathrm{i}-\mathrm{k}}, \mathrm{v}_{2 \mathrm{r}+3-\mathrm{i}-\mathrm{k}}, \ldots, \mathrm{v}_{2 \mathrm{r}}\right\}$.

Thus,

$\left|\mathrm{S}_{1}\left(\mathrm{v}_{\mathrm{i}}, \mathrm{k}\right)\right|=2,\left|\mathrm{~S}_{2}\left(\mathrm{v}_{\mathrm{i}}, \mathrm{k}\right)\right|=\mathrm{t}+1-2 \mathrm{k}-2 \mathrm{i}$.

So, by Lemma 1.1, we have, for $\mathrm{i}=1,2, \ldots, \mathrm{k}$,

$\mathrm{C}_{\mathrm{n}}\left(\mathrm{v}_{\mathrm{i}}, \mathrm{P}_{\mathrm{t}}^{2}, \mathrm{k}\right)=\left(\begin{array}{c}\mathrm{t}+3-2 \mathrm{k}-2 \mathrm{i} \\ \mathrm{n}-1\end{array}\right)-\left(\begin{array}{c}\mathrm{t}+1-2 \mathrm{k}-2 \mathrm{i} \\ \mathrm{n}-1\end{array}\right)$.

(2). For $\mathrm{i}=1,2, \ldots, \mathrm{k}-1$, we obtain, from Fig. 2.2,

$\mathrm{S}_{1}\left(\mathrm{v}_{\mathrm{r}+1-\mathrm{i}}, \mathrm{k}\right)=\left\{\mathrm{v}_{\mathrm{r}-\mathrm{k}-\mathrm{i}+1}, \mathrm{v}_{\mathrm{r}+\mathrm{k}+\mathrm{i}}\right\}$,

$\mathrm{S}_{2}\left(\mathrm{v}_{\mathrm{r}+1-\mathrm{i}}, \mathrm{k}\right)=\mathrm{V}\left(\mathrm{P}_{\mathrm{t}}^{2}\right)-\left\{\mathrm{v}_{\mathrm{r}-\mathrm{k}-\mathrm{i}+1}, \mathrm{v}_{\mathrm{r}-\mathrm{k}-\mathrm{i}+2}, \ldots, \mathrm{v}_{\mathrm{r}}, \mathrm{v}_{\mathrm{r}+1}, \ldots, \mathrm{v}_{\mathrm{r}+\mathrm{k}+\mathrm{i}}\right\}$.

Thus,

$\left|\mathrm{S}_{1}\left(\mathrm{v}_{\mathrm{r}+1-\mathrm{i}}, \mathrm{k}\right)\right|=2,\left|\mathrm{~S}_{2}\left(\mathrm{v}_{\mathrm{r}+1-\mathrm{i}}, \mathrm{k}\right)\right|=\mathrm{t}-2 \mathrm{k}-2 \mathrm{i}$.

So, using Lemma 1.1 , we obtain , for $\mathrm{i}=1,2, \ldots, \mathrm{k}-1$,

$\mathrm{C}_{\mathrm{n}}\left(\mathrm{v}_{\mathrm{r}+1-\mathrm{i}}, \mathrm{P}_{\mathrm{t}}^{2}, \mathrm{k}\right)=\left(\begin{array}{c}\mathrm{t}+2-2 \mathrm{k}-2 \mathrm{i} \\ \mathrm{n}-1\end{array}\right)-\left(\begin{array}{c}\mathrm{t}-2 \mathrm{k}-2 \mathrm{i} \\ \mathrm{n}-1\end{array}\right)$.

(3). For $v_{r-k+1}$, we have

$\mathrm{S}_{1}\left(\mathrm{v}_{\mathrm{r}-\mathrm{k}+1}, \mathrm{k}\right)=\left\{\mathrm{v}_{\mathrm{r}+1}, \mathrm{v}_{2 \mathrm{k}+\mathrm{r}}, \mathrm{v}_{\mathrm{r}+1-2 \mathrm{k}}\right\}$,

$\mathrm{S}_{2}\left(\mathrm{v}_{\mathrm{r}-\mathrm{k}+1}, \mathrm{k}\right)=\mathrm{V}\left(\mathrm{P}_{\mathrm{t}}^{2}\right)-\left\{\mathrm{v}_{\mathrm{r}-2 \mathrm{k}+1}, \mathrm{v}_{\mathrm{r}-2 \mathrm{k}+2}, \ldots, \mathrm{v}_{\mathrm{r}}, \mathrm{v}_{\mathrm{r}+1}, \ldots, \mathrm{v}_{\mathrm{r}+2 \mathrm{k}}\right\}$.

Thus,

$\left|\mathrm{S}_{1}\left(\mathrm{v}_{\mathrm{r}-\mathrm{k}+1}, \mathrm{k}\right)\right|=3,\left|\mathrm{~S}_{2}\left(\mathrm{v}_{\mathrm{r}-\mathrm{k}+1}, \mathrm{k}\right)\right|=\mathrm{t}-4 \mathrm{k}$.

So , using Lemma 1.1 , we get ,

$\mathrm{C}_{\mathrm{n}}\left(\mathrm{v}_{\mathrm{r}-\mathrm{k}+1}, \mathrm{P}_{\mathrm{t}}^{2}, \mathrm{k}\right)=\left(\begin{array}{c}\mathrm{t}+3-4 \mathrm{k} \\ \mathrm{n}-1\end{array}\right)-\left(\begin{array}{c}\mathrm{t}-4 \mathrm{k} \\ \mathrm{n}-1\end{array}\right)$.

(4). For $\mathrm{i}=\mathrm{k}+1, \mathrm{k}+2, \ldots, \mathrm{r}-\mathrm{k}$,

$\mathrm{S}_{1}\left(\mathrm{v}_{\mathrm{i}}, \mathrm{k}\right)=\left\{\mathrm{v}_{\mathrm{i}-\mathrm{k}}, \mathrm{v}_{\mathrm{i}+\mathrm{k}}, \mathrm{v}_{2 \mathrm{r}+\mathrm{k}-\mathrm{i}+1}, \mathrm{v}_{2 \mathrm{r}-\mathrm{k}-\mathrm{i}+2}\right\}$,

$\mathrm{S}_{2}\left(\mathrm{v}_{\mathrm{i}}, \mathrm{k}\right)=\mathrm{V}\left(\mathrm{P}_{\mathrm{t}}^{2}\right)-\left\{\mathrm{v}_{\mathrm{i}-\mathrm{k}}, \mathrm{v}_{\mathrm{i}-\mathrm{k}+1}, \ldots, \mathrm{v}_{\mathrm{i}+\mathrm{k}}, \mathrm{v}_{2 \mathrm{r}-\mathrm{k}-\mathrm{i}+2}, \mathrm{v}_{2 \mathrm{r}-\mathrm{k}-\mathrm{i}+3}, \ldots, \mathrm{v}_{2 \mathrm{r}+\mathrm{k}-\mathrm{i}+1}\right\}$.

Thus,

$\left|\mathrm{S}_{1}\left(\mathrm{v}_{\mathrm{i}}, \mathrm{k}\right)\right|=4,\left|\mathrm{~S}_{2}\left(\mathrm{v}_{\mathrm{i}}, \mathrm{k}\right)\right|=\mathrm{t}-4 \mathrm{k}-1$.

Therefore, using Lemma 1.1, we get, for $\mathrm{i}=\mathrm{k}+1, \mathrm{k}+2, \ldots, \mathrm{r}-\mathrm{k}$,

$\mathrm{C}_{\mathrm{n}}\left(\mathrm{v}_{\mathrm{i}}, \mathrm{P}_{\mathrm{t}}^{2}, \mathrm{k}\right)=\left(\begin{array}{c}\mathrm{t}-4 \mathrm{k}+3 \\ \mathrm{n}-1\end{array}\right)-\left(\begin{array}{c}\mathrm{t}-4 \mathrm{k}-1 \\ \mathrm{n}-1\end{array}\right)$.

Thus, from (2.2.6) and summing up the formulas (c1)-(c4) we get for $2 \leq \mathrm{k} \leq\left\lfloor\frac{\delta_{\mathrm{n}}}{2}\right\rfloor$, 


$$
\begin{aligned}
\mathrm{C}_{\mathrm{n}}\left(\mathrm{P}_{\mathrm{t}}^{2}, \mathrm{k}\right) & =2\left\{\sum_{\mathrm{i}=1}^{\mathrm{k}}\left[\left(\begin{array}{c}
\mathrm{t}+3-2 \mathrm{k}-2 \mathrm{i} \\
\mathrm{n}-1
\end{array}\right)-\left(\begin{array}{c}
\mathrm{t}+1-2 \mathrm{k}-2 \mathrm{i} \\
\mathrm{n}-1
\end{array}\right)\right]\right. \\
& +\sum_{\mathrm{i}=1}^{\mathrm{k}-1}\left[\left(\begin{array}{c}
\mathrm{t}+2-2 \mathrm{k}-2 \mathrm{i} \\
\mathrm{n}-1
\end{array}\right)-\left(\begin{array}{c}
\mathrm{t}-2 \mathrm{k}-2 \mathrm{i} \\
\mathrm{n}-1
\end{array}\right)\right] \\
& \left.+\left(\begin{array}{c}
\mathrm{t}-4 \mathrm{k}+3 \\
\mathrm{n}-1
\end{array}\right)-\left(\begin{array}{c}
\mathrm{t}-4 \mathrm{k} \\
\mathrm{n}-1
\end{array}\right)+(\mathrm{r}-2 \mathrm{k})\left[\left(\begin{array}{c}
\mathrm{t}-4 \mathrm{k}+3 \\
\mathrm{n}-1
\end{array}\right)-\left(\begin{array}{c}
\mathrm{t}-4 \mathrm{k}-1 \\
\mathrm{n}-1
\end{array}\right)\right]\right\} . \\
& =2\left\{\left[\left(\begin{array}{c}
\mathrm{t}-2 \mathrm{k}+1 \\
\mathrm{n}-1
\end{array}\right)-\left(\begin{array}{c}
\mathrm{t}-4 \mathrm{k}+1 \\
\mathrm{n}-1
\end{array}\right)\right]+\left[\left(\begin{array}{c}
\mathrm{t}-2 \mathrm{k} \\
\mathrm{n}-1
\end{array}\right)-\left(\begin{array}{c}
\mathrm{t}-4 \mathrm{k}+2 \\
\mathrm{n}-1
\end{array}\right)\right]\right. \\
& \left.+\left(\begin{array}{c}
\mathrm{t}-4 \mathrm{k}+3 \\
\mathrm{n}-1
\end{array}\right)-\left(\begin{array}{c}
\mathrm{t}-4 \mathrm{k} \\
\mathrm{n}-1
\end{array}\right)+(\mathrm{r}-2 \mathrm{k})\left[\left(\begin{array}{c}
\mathrm{t}-4 \mathrm{k}+3 \\
\mathrm{n}-1
\end{array}\right)-\left(\begin{array}{c}
\mathrm{t}-4 \mathrm{k}-1 \\
\mathrm{n}-1
\end{array}\right)\right]\right\} . \\
& =2\left[\left(\begin{array}{c}
\mathrm{t}-2 \mathrm{k} \\
\mathrm{n}-1
\end{array}\right)+\left(\begin{array}{c}
\mathrm{t}-2 \mathrm{k}+1 \\
\mathrm{n}-1
\end{array}\right)\right]+(\mathrm{t}-4 \mathrm{k}+2)\left(\begin{array}{c}
\mathrm{t}-4 \mathrm{k}+3 \\
\mathrm{n}-1
\end{array}\right) \\
& -2 \sum_{\mathrm{j}=0}^{2}\left(\begin{array}{c}
\mathrm{t}-4 \mathrm{k}+\mathrm{j})-(\mathrm{t}-4 \mathrm{k})\left(\begin{array}{c}
\mathrm{t}-4 \mathrm{k}-1 \\
\mathrm{n}-1
\end{array}\right) .
\end{array}\right.
\end{aligned}
$$

Now, we give the proof of (2.2.4) for $\left\lfloor\frac{\delta_{n}}{2}\right\rfloor+1 \leq k \leq \delta_{n}$. Here, we have two cases:

(a). For $\mathrm{i}=1,2, \ldots, \mathrm{r}-\mathrm{k}$,

$\mathrm{S}_{1}\left(\mathrm{v}_{\mathrm{i}}, \mathrm{k}\right)=\left\{\mathrm{v}_{\mathrm{i}+\mathrm{k}}, \mathrm{v}_{2 \mathrm{r}+2-\mathrm{i}-\mathrm{k}}\right\}$,

$\mathrm{S}_{2}\left(\mathrm{v}_{\mathrm{i}}, \mathrm{k}\right)=\mathrm{V}\left(\mathrm{P}_{\mathrm{t}}^{2}\right)-\left\{\mathrm{v}_{1}, \mathrm{v}_{2}, \ldots, \mathrm{v}_{\mathrm{i}+\mathrm{k}}, \mathrm{v}_{2 \mathrm{r}+2-\mathrm{i}-\mathrm{k}}, \mathrm{v}_{2 \mathrm{r}+3-\mathrm{i}-\mathrm{k}}, \ldots, \mathrm{v}_{2 \mathrm{r}}\right\}$.

Thus,

$\left|\mathrm{S}_{1}\left(\mathrm{v}_{\mathrm{i}}, \mathrm{k}\right)\right|=2,\left|\mathrm{~S}_{2}\left(\mathrm{v}_{\mathrm{i}}, \mathrm{k}\right)\right|=\mathrm{t}+1-2 \mathrm{k}-2 \mathrm{i}$.

So, by Lemma 1.1, we have, for $\mathrm{i}=1,2, \ldots, \mathrm{r}-\mathrm{k}$,

$\mathrm{C}_{\mathrm{n}}\left(\mathrm{v}_{\mathrm{i}}, \mathrm{P}_{\mathrm{t}}^{2}, \mathrm{k}\right)=\left(\begin{array}{c}\mathrm{t}-2 \mathrm{k}-2 \mathrm{i}+3 \\ \mathrm{n}-1\end{array}\right)-\left(\begin{array}{c}\mathrm{t}-2 \mathrm{k}-2 \mathrm{i}+1 \\ \mathrm{n}-1\end{array}\right)$.

(b). For $\mathrm{v}_{\mathrm{r}+1-\mathrm{i}}, \mathrm{i}=1,2, \ldots, \mathrm{r}-\mathrm{k}$, we have

$\mathrm{S}_{1}\left(\mathrm{v}_{\mathrm{r}+1-\mathrm{i}}, \mathrm{k}\right)=\left\{\mathrm{v}_{\mathrm{r}-\mathrm{k}-\mathrm{i}+1}, \mathrm{v}_{\mathrm{r}+\mathrm{k}+\mathrm{i}}\right\}$,

$\mathrm{S}_{2}\left(\mathrm{v}_{\mathrm{r}+\mathrm{l}-\mathrm{i}}, \mathrm{k}\right)=\mathrm{V}\left(\mathrm{P}_{\mathrm{t}}^{2}\right)-\left\{\mathrm{v}_{\mathrm{r}-\mathrm{k}-\mathrm{i}+1}, \mathrm{v}_{\mathrm{r}-\mathrm{k}-\mathrm{i}+2}, \ldots, \mathrm{v}_{\mathrm{r}}, \mathrm{v}_{\mathrm{r}+1}, \ldots, \mathrm{v}_{\mathrm{r}+\mathrm{k}+\mathrm{i}}\right\}$.

Thus,

$\left|\mathrm{S}_{1}\left(\mathrm{v}_{\mathrm{r}+1-\mathrm{i}}, \mathrm{k}\right)\right|=2,\left|\mathrm{~S}_{2}\left(\mathrm{v}_{\mathrm{r}+1-\mathrm{i}}, \mathrm{k}\right)\right|=\mathrm{t}-2 \mathrm{k}-2 \mathrm{i}$.

So, by Lemma 1.1 , we have, for $\mathrm{i}=1,2, \ldots, \mathrm{r}-\mathrm{k}$,

$\mathrm{C}_{\mathrm{n}}\left(\mathrm{v}_{\mathrm{r}+1-\mathrm{i}}, \mathrm{P}_{\mathrm{t}}^{2}, \mathrm{k}\right)=\left(\begin{array}{c}\mathrm{t}-2 \mathrm{k}-2 \mathrm{i}+2 \\ \mathrm{n}-1\end{array}\right)-\left(\begin{array}{c}\mathrm{t}-2 \mathrm{k}-2 \mathrm{i} \\ \mathrm{n}-1\end{array}\right)$.

Therefore, using (2.2.6) and summing up (d1) and (d2), we get for $\left\lfloor\frac{\delta_{\mathrm{n}}}{2}\right\rfloor+1 \leq \mathrm{k} \leq \delta_{\mathrm{n}}$, 


$$
\begin{aligned}
\mathrm{C}_{\mathrm{n}}\left(\mathrm{P}_{\mathrm{t}}^{2}, \mathrm{k}\right) & =2\left\{\sum_{\mathrm{i}=1}^{\mathrm{r}-\mathrm{k}}\left[\left(\begin{array}{c}
\mathrm{t}-2 \mathrm{k}-2 \mathrm{i}+3 \\
\mathrm{n}-1
\end{array}\right)-\left(\begin{array}{c}
\mathrm{t}-2 \mathrm{k}-2 \mathrm{i}+1 \\
\mathrm{n}-1
\end{array}\right)\right]\right. \\
& \left.+\sum_{\mathrm{i}=1}^{\mathrm{r}-\mathrm{k}}\left[\left(\begin{array}{c}
\mathrm{t}-2 \mathrm{k}-2 \mathrm{i}+2 \\
\mathrm{n}-1
\end{array}\right)-\left(\begin{array}{c}
\mathrm{t}-2 \mathrm{k}-2 \mathrm{i} \\
\mathrm{n}-1
\end{array}\right)\right]\right\} \\
& =2\left\{\left[\left(\begin{array}{c}
\mathrm{t}-2 \mathrm{k}+1 \\
\mathrm{n}-1
\end{array}\right)-\left(\begin{array}{c}
\mathrm{t}-2 \mathrm{r}+1 \\
\mathrm{n}-1
\end{array}\right)\right]+\left[\left(\begin{array}{c}
\mathrm{t}-2 \mathrm{k} \\
\mathrm{n}-1
\end{array}\right)-\left(\begin{array}{c}
\mathrm{t}-2 \mathrm{r} \\
\mathrm{n}-1
\end{array}\right)\right]\right\} \\
& =2\left[\left(\begin{array}{c}
\mathrm{t}-2 \mathrm{k}+1 \\
\mathrm{n}-1
\end{array}\right)+\left(\begin{array}{c}
\mathrm{t}-2 \mathrm{k} \\
\mathrm{n}-1
\end{array}\right)\right], \text { because } \mathrm{n} \geq 3 .
\end{aligned}
$$

Second, the proofs of (2.2.3) and (2.2.4) for odd $\mathbf{t}, \mathrm{t}=2 \mathrm{r}+1, \mathrm{r} \geq 3$, are similar to the proofs of (2.2.3) and (2.2.4) for even $t$.

Hence, the proof of the Theorem is completed.

\#

Corollary 2.3: The $n-W i e n e r$ index of $\mathrm{P}_{t}^{2}$ is given by:

$\mathrm{W}_{\mathrm{n}}\left(\mathrm{P}_{\mathrm{t}}^{2}\right)=\mathrm{t}\left(\begin{array}{l}\mathrm{t}-1 \\ \mathrm{n}-1\end{array}\right)-2\left[\left(\begin{array}{c}\mathrm{t}-3 \\ \mathrm{n}-1\end{array}\right)+\left(\begin{array}{c}\mathrm{t}-4 \\ \mathrm{n}-1\end{array}\right)\right]-(\mathrm{t}-4)\left(\begin{array}{c}\mathrm{t}-5 \\ \mathrm{n}-1\end{array}\right)+\sum_{\mathrm{k}=2}^{\delta_{\mathrm{n}}} \mathrm{kC}_{\mathrm{n}}\left(\mathrm{P}_{\mathrm{t}}^{2}, \mathrm{k}\right)$

in which

$$
\begin{aligned}
& \mathrm{C}_{\mathrm{n}}\left(\mathrm{P}_{\mathrm{t}}^{2}, \mathrm{k}\right)=2\left[\left(\begin{array}{c}
\mathrm{t}-2 \mathrm{k} \\
\mathrm{n}-1
\end{array}\right)+\left(\begin{array}{c}
\mathrm{t}-2 \mathrm{k}+1 \\
\mathrm{n}-1
\end{array}\right)\right]+(\mathrm{t}-4 \mathrm{~m}+2)\left(\begin{array}{c}
\mathrm{t}-4 \mathrm{k}+3 \\
\mathrm{n}-1
\end{array}\right) \\
&-2 \sum_{\mathrm{i}=0}^{2}\left(\begin{array}{c}
\mathrm{t}-4 \mathrm{k}+\mathrm{i} \\
\mathrm{n}-1
\end{array}\right)-(\mathrm{t}-4 \mathrm{k})\left(\begin{array}{c}
\mathrm{t}-4 \mathrm{k}-1 \\
\mathrm{n}-1
\end{array}\right), 2 \leq \mathrm{k} \leq\left\lfloor\frac{\delta_{\mathrm{n}}}{2}\right\rfloor, \\
& \mathrm{C}_{\mathrm{n}}\left(\mathrm{P}_{\mathrm{t}}^{2}, \mathrm{k}\right)=2\left[\left(\begin{array}{c}
\mathrm{t}-2 \mathrm{k} \\
\mathrm{n}-1
\end{array}\right)+\left(\begin{array}{c}
\mathrm{t}-2 \mathrm{k}+1 \\
\mathrm{n}-1
\end{array}\right)\right],\left\lfloor\frac{\delta_{\mathrm{n}}}{2}\right\rfloor+1 \leq \mathrm{k} \leq \delta_{\mathrm{n}} . \quad \#
\end{aligned}
$$

\section{The n-Hosoya Polynomial of the Square of a Cycle :}

There are many classes of connected graphs $G$ in which for each $k, 1 \leq k \leq \delta_{n}$, $C_{n}(v, G, k)$ is the same for every vertex $v \in V(G)$; such graphs are called [2] vertex-ndistance regular graphs, and for the given value of $\mathrm{n}, 2 \leq \mathrm{n} \leq \mathrm{t}$, $\mathrm{H}_{\mathrm{n}}(\mathrm{G} ; \mathrm{x})=\mathrm{tH}_{\mathrm{n}}(\mathrm{v}, \mathrm{G} ; \mathrm{x})$, where $\mathrm{v}$ is any vertex of $\mathrm{G}$ and $\mathrm{t}$ is the order of $\mathrm{G}$.

The graph $C_{t}^{2}$ is the square of a cycle of order $t$, shown in Fig. 3.1. We shall find the ndiameter, n-Hosoya polynomial, and $n-W i e n e r$ index of $C_{t}^{2}$. 


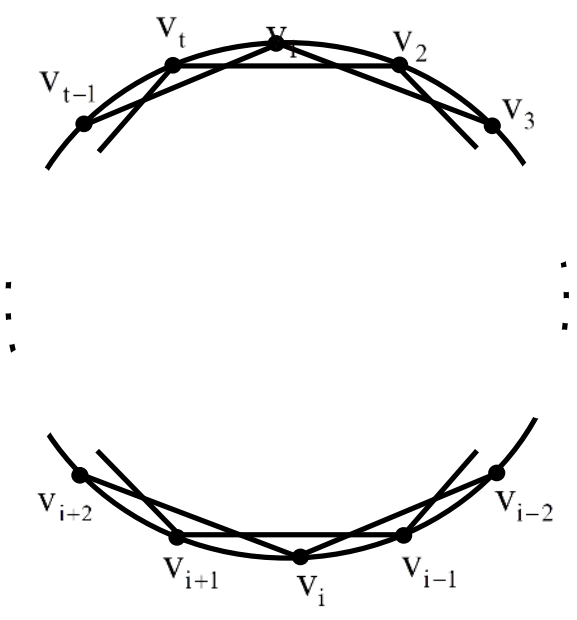

Fig. (3.1) The Cycle Square $C_{t}^{2}, \mathrm{t} \geq 6$.

Lemma 3.1: $\operatorname{diam}_{n}\left(C_{t}^{2}\right)=\delta_{n}=1+\left\lfloor\frac{t-n}{4}\right\rfloor, n \geq 2, t \geq 6$.

Proof: Let $\mathrm{m}=\left\lfloor\frac{\mathrm{t}}{4}\right\rfloor$, then $\mathrm{t}=4 \mathrm{~m}+\mathrm{r}, \mathrm{r}=0,1,2,3$.

For $r=2, C_{t}^{2}$ is redrawn in Fig. 3.2.

Since, $C_{t}^{2}$ is vertex n-distance regular graph, then $\operatorname{diam}_{n}\left(C_{t}^{2}\right)=e_{n}\left(v_{1}\right)$.

To find the n-eccentricity of $\mathrm{v}_{1}$, we partition $\mathrm{V}\left(\mathrm{C}_{\mathrm{t}}^{2}\right)-\left\{\mathrm{v}_{1}\right\}$ into $\mathrm{S}_{1}, \mathrm{~S}_{2}, \ldots, \mathrm{S}_{\mathrm{m}+1}$, where $\mathrm{S}_{1}=\left\{\mathrm{v}_{2}, \mathrm{v}_{3}, \mathrm{v}_{\mathrm{t}}, \mathrm{v}_{\mathrm{t}-1}\right\}$,

$\mathrm{S}_{2}=\left\{\mathrm{v}_{4}, \mathrm{v}_{5}, \mathrm{v}_{\mathrm{t}-2}, \mathrm{v}_{\mathrm{t}-3}\right\}$,

$\mathrm{S}_{3}=\left\{\mathrm{v}_{6}, \mathrm{v}_{7}, \mathrm{v}_{\mathrm{t}-4}, \mathrm{v}_{\mathrm{t}-5}\right\}$,

$\mathrm{S}_{\mathrm{j}}=\left\{\mathrm{v}_{2 \mathrm{j}}, \mathrm{v}_{2 \mathrm{j}+1}, \mathrm{v}_{\mathrm{t}-2(\mathrm{j}-1)}, \mathrm{v}_{\mathrm{t}-2 \mathrm{j}+1}\right\}$,

$\mathrm{S}_{\mathrm{m}}=\left\{\mathrm{v}_{2 \mathrm{~m}}, \mathrm{v}_{2 \mathrm{~m}+1}, \mathrm{v}_{\mathrm{t}-2 \mathrm{~m}+2}, \mathrm{v}_{\mathrm{t}-2 \mathrm{~m}+1}\right\}$,

$\mathrm{S}_{\mathrm{m}+1}=\mathrm{V}\left(\mathrm{C}_{\mathrm{t}}^{2}\right)-\left(\bigcup_{\mathrm{j}=1}^{\mathrm{m}} \mathrm{S}_{\mathrm{j}} \cup\left\{\mathrm{v}_{1}\right\}\right)$. 

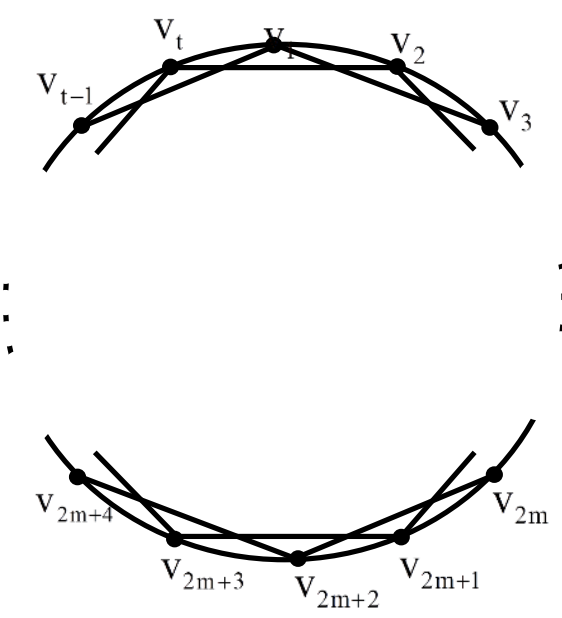

Fig. (3.2). The Cycle Square $C_{t}^{2}, t=4 m+2, m \geq 1$.

It is clear that each vertex of $\mathrm{S}_{\mathrm{j}}, 1 \leq \mathrm{j} \leq \mathrm{m}$, is of (standard) distance $\mathrm{j}$ from $\mathrm{v}_{1}$; and each of the other vertices (if exists) of $\mathrm{C}_{t}^{2}$ (here in Fig. 3.2, we have $\left\{\mathrm{v}_{\frac{\mathrm{t}}{2}+1}\right\}=\left\{\mathrm{v}_{2 \mathrm{~m}+2}\right\}=\mathrm{S}_{\mathrm{m}+1}$ ) is of the distance $\mathrm{m}+1$ from $\mathrm{v}_{1}$. Notice that if $\mathrm{t}=4 \mathrm{~m}+1$, then $S_{m+1}$ is empty, and if $t=4 m$ then, $S_{m+1}$ is empty and $S_{m}$ consists of three elements; if $\mathrm{t}=4 \mathrm{~m}+2, \mathrm{t}=4 \mathrm{~m}+3$, then $\mathrm{S}_{\mathrm{m}+1}$ consists of one, respectively two, elements.

Let $\mathrm{k}$ be the greatest positive integer such that the set $\bigcup_{\mathrm{i}=\mathrm{k}}^{\mathrm{m}+1} \mathrm{~S}_{\mathrm{i}}$ consists of at least $(\mathrm{n}-1)$ vertices. Therefore, since $\left|S_{i}\right| \leq 4$.

$4(k-1)+1+(n-1) \leq t$,

$4 \mathrm{k} \leq \mathrm{t}-\mathrm{n}+4$,

$\mathrm{k} \leq \frac{\mathrm{t}-\mathrm{n}}{4}+1$

Therefore, $\operatorname{diam}_{\mathrm{n}}\left(\mathrm{C}_{\mathrm{t}}^{2}\right)=\mathrm{k}=1+\left\lfloor\frac{\mathrm{t}-\mathrm{n}}{4}\right\rfloor,(\because \mathrm{k}$ is positive integer $)$. \#

Theorem 3.2: For any $n \geq 3$, the $n$-Hosoya polynomial of $C_{t}^{2}, t \geq 6$ is given by:

$$
\mathrm{H}_{\mathrm{n}}\left(\mathrm{C}_{\mathrm{t}}^{2} ; \mathrm{x}\right)=\mathrm{t}\left(\begin{array}{c}
\mathrm{t}-1 \\
\mathrm{n}-2
\end{array}\right)+\sum_{\mathrm{k}=1}^{\delta_{\mathrm{n}}-1} \mathrm{t}\left(\left(\begin{array}{c}
\mathrm{t}-4 \mathrm{k}+3 \\
\mathrm{n}-1
\end{array}\right)-\left(\begin{array}{c}
\mathrm{t}-4 \mathrm{k}-1 \\
\mathrm{n}-1
\end{array}\right)\right] \mathrm{x}^{\mathrm{k}}+\mathrm{C}_{\mathrm{n}}\left(\mathrm{C}_{\mathrm{t}}^{2}, \delta_{\mathrm{n}}\right) \mathrm{x}^{\delta_{\mathrm{n}}},
$$

Where, $C_{n}\left(C_{t}^{2}, \delta_{n}\right)$ is determined in Remark 3.3, and $\delta_{n}$ is determined by Lemma 3.1.

Proof: Let $S$ be a set of (n-1) vertices of $V\left(C_{t}^{2}\right)$ such that $v_{1} \notin S, v_{1} \in V\left(C_{t}^{2}\right)$ and $\mathrm{d}_{\mathrm{n}}\left(\mathrm{v}_{1}, \mathrm{~S}\right)=\mathrm{k}, 2 \leq \mathrm{k} \leq \delta_{\mathrm{n}}-1$. Hence, $\mathrm{S}$ does not contain any vertex from $\left\{\mathrm{v}_{\mathrm{t}-2 \mathrm{k}+3}, \ldots, \mathrm{v}_{\mathrm{t}-1}, \mathrm{v}_{\mathrm{t}}, \mathrm{v}_{1}, \mathrm{v}_{2}, \mathrm{v}_{3}, \ldots, \mathrm{v}_{2 \mathrm{k}-1}\right\}$, (see Fig. 3.1), but $\mathrm{S}$ must contain , at least, 
one vertex of $\left\{\mathrm{v}_{2 \mathrm{k}}, \mathrm{v}_{2 \mathrm{k}+1}, \mathrm{v}_{\mathrm{t}-2 \mathrm{k}+2}, \mathrm{v}_{\mathrm{t}-2 \mathrm{k}+1}\right\}$. Then, the number of vertices in $\mathrm{C}_{\mathrm{t}}^{2}$ of distance more than $\mathrm{k}$ from $\mathrm{v}_{1}$ is $(\mathrm{t}-4 \mathrm{k}-1)$ and there are four vertices in $\mathrm{C}_{\mathrm{t}}^{2}$ of distance $\mathrm{k}$ from $\mathrm{v}_{1}$. Hence, by Lemma 1.1 , $\mathrm{C}_{\mathrm{n}}\left(\mathrm{v}_{1}, \mathrm{C}_{\mathrm{t}}^{2}, \mathrm{k}\right)=\left(\begin{array}{c}\mathrm{t}-4 \mathrm{k}+3 \\ \mathrm{n}-1\end{array}\right)-\left(\begin{array}{c}\mathrm{t}-4 \mathrm{k}-1 \\ \mathrm{n}-1\end{array}\right)$, for $2 \leq \mathrm{k} \leq \delta_{\mathrm{n}}-1$.

Moreover, it is clear that

$$
\mathrm{C}_{\mathrm{n}}\left(\mathrm{v}_{1}, \mathrm{C}_{\mathrm{t}}^{2}, 1\right)==\left(\begin{array}{c}
\mathrm{t}-1 \\
\mathrm{n}-1
\end{array}\right)-\left(\begin{array}{c}
\mathrm{t}-5 \\
\mathrm{n}-1
\end{array}\right) \text {. }
$$

Since $C_{n}\left(v_{1}, C_{t}^{2}, k\right)=C_{n}\left(v_{i}, C_{t}^{2}, k\right), 2 \leq i \leq t$, then $\mathrm{C}_{\mathrm{n}}\left(\mathrm{C}_{\mathrm{t}}^{2}, \mathrm{k}\right)=\mathrm{t}\left[\left(\begin{array}{c}\mathrm{t}-4 \mathrm{k}+3 \\ \mathrm{n}-1\end{array}\right)-\left(\begin{array}{c}\mathrm{t}-4 \mathrm{k}-1 \\ \mathrm{n}-1\end{array}\right)\right]$, for $1 \leq \mathrm{k} \leq \delta_{\mathrm{n}}-1 . \quad$ \#

Remark 3.3: From Fig. 3-2, we can easily obtain $C_{n}\left(C_{t}^{2}, \delta_{n}\right)$, for $n \geq 3$.

1. If $\mathrm{t}=4 \mathrm{~m}+3$, then,

$$
\mathrm{C}_{\mathrm{n}}\left(\mathrm{C}_{\mathrm{t}}^{2}, \delta_{\mathrm{n}}\right)=\left\{\begin{array}{l}
\mathrm{t} ; \mathrm{n}=3 \\
\mathrm{t}\left[\left(\begin{array}{c}
\mathrm{t}-4 \delta_{\mathrm{n}}+3 \\
\mathrm{n}-1
\end{array}\right)-\left(\begin{array}{c}
\mathrm{t}-4 \delta_{\mathrm{n}}-1 \\
\mathrm{n}-1
\end{array}\right)\right] ; \mathrm{n} \geq 4 .
\end{array}\right.
$$

2. If $\mathrm{t}=4 \mathrm{~m}+2,4 \mathrm{~m}+1$, then,

$$
\mathrm{C}_{\mathrm{n}}\left(\mathrm{C}_{\mathrm{t}}^{2}, \delta_{\mathrm{n}}\right)=\mathrm{t}\left[\left(\begin{array}{c}
\mathrm{t}-4 \delta_{\mathrm{n}}+3 \\
\mathrm{n}-1
\end{array}\right)-\left(\begin{array}{c}
\mathrm{t}-4 \delta_{\mathrm{n}}-1 \\
\mathrm{n}-1
\end{array}\right)\right] ; \mathrm{n} \geq 3 .
$$

3. If $\mathrm{t}=4 \mathrm{~m}$, then,

$$
\mathrm{C}_{\mathrm{n}}\left(\mathrm{C}_{\mathrm{t}}^{2}, \delta_{\mathrm{n}}\right)=\left\{\begin{array}{l}
\mathrm{t}\left(\begin{array}{c}
3 \\
\mathrm{n}-1
\end{array}\right) ; \mathrm{n}=3,4 \\
\left.\mathrm{t}\left(\begin{array}{c}
\mathrm{t}-4 \delta_{\mathrm{n}}+3 \\
\mathrm{n}-1
\end{array}\right)-\left(\begin{array}{c}
\mathrm{t}-4 \delta_{\mathrm{n}}-1 \\
\mathrm{n}-1
\end{array}\right)\right] ; \mathrm{n} \geq 5 .
\end{array}\right.
$$

Corollary 3.4: The $n-W i e n e r$ index of $C_{t}^{2}$ is given by:

$\mathrm{W}_{\mathrm{n}}\left(\mathrm{C}_{\mathrm{t}}^{2}\right)=\sum_{\mathrm{k}=1}^{\delta_{\mathrm{n}}} \mathrm{kC}_{\mathrm{n}}\left(\mathrm{C}_{\mathrm{t}}^{2}, \mathrm{k}\right)$, where $\mathrm{C}_{\mathrm{n}}\left(\mathrm{C}_{\mathrm{t}}^{2}, \mathrm{k}\right), 1 \leq \mathrm{k} \leq \delta_{\mathrm{n}}$ is given in Theorem 3.2 and Remark 3.3 . \# 


\section{REFERENCES}

[1] Ahmed, H.G. (2007), "On Wiener Polynomials of n-Distance in Graphs", M.Sc. Thesis, University of Dohuk, Dohuk, Iraq.

[2] Ali, A.A. and Ali, A.M.; (2006), "Wiener Polynomials of the Generalized Distance for some Special Graphs", Raf. J. Comp. Sc. And Maths. Vol.3, No.2, pp.103-120.

[3] Ali, A.A. and Ali, A.M.; (2010), "The n-Hosoya Polynomials of some Classes of Thorn Graphs", Raf. J. Comp. Sc. And Maths. Vol.7, No.1, pp.81-97.

[4] Ali, A.A. and Ahmed, H.G.; (2012), "The n-Hosoya Polynomial of $\mathrm{W}_{\alpha} \otimes \mathrm{C}_{\beta}$ ", Raf. J. Comp. Sc. and Maths. Vol.9, No.2, pp.139-150.

[5] Buckley, F. and Harary, F.; (1990), Distance in Graphs. Addison -Wesley, Redwood, California.

[6] Diestel, R; (2000), Graphs Theory, electronic ed., Springer-Verlag, New York.

[7] Harary, F. and Ross, I.C.; (1960), "The square of a tree", Bell Syst. Tech. J., 39, p. 641-647. 